\title{
Dissecting the signaling pathways associated with the oncogenic activity of MLK3 P252H mutation
}

\author{
Sérgia Velho ${ }^{1}$, Ana Pinto ${ }^{1,2}$, Danilo Licastro ${ }^{3}$, Maria José Oliveira ${ }^{2}$, Filipa Sousa ${ }^{1}$, Elia Stupka ${ }^{4}$ and Raquel Seruca ${ }^{1,5^{*}}$
}

\begin{abstract}
Background: MLK3 gene mutations were described to occur in about $20 \%$ of microsatellite unstable gastrointestinal cancers and to harbor oncogenic activity. In particular, mutation P252H, located in the kinase domain, was found to have a strong transforming potential, and to promote the growth of highly invasive tumors when subcutaneously injected in nude mice. Nevertheless, the molecular mechanism underlying the oncogenic activity of $\mathrm{P} 252 \mathrm{H}$ mutant remained elusive.
\end{abstract}

Methods: In this work, we performed Illumina Whole Genome arrays on three biological replicas of human HEK293 cells stably transfected with the wild-type MLK3, the P252H mutation and with the empty vector (Mock) in order to identify the putative signaling pathways associated with $\mathrm{P} 252 \mathrm{H}$ mutation.

Results: Our microarray results showed that mutant MLK3 deregulates several important colorectal cancer- associated signaling pathways such as WNT, MAPK, NOTCH, TGF-beta and p53, helping to narrow down the number of potential MLK3 targets responsible for its oncogenic effects. A more detailed analysis of the alterations affecting the WNT signaling pathway revealed a down-regulation of molecules involved in the canonical pathway, such as DVL2, LEF1, CCND1 and c-Myc, and an up-regulation of DKK, a well-known negative regulator of canonical WNT signaling, in MLK3 mutant cells. Additionally, FZD6 and FZD10 genes, known to act as negative regulators of the canonical WNT signaling cascade and as positive regulators of the planar cell polarity (PCP) pathway, a non-canonic WNT pathway, were found to be up-regulated in $\mathrm{P} 252 \mathrm{H}$ cells.

Conclusion: The results provide an overall view of the expression profile associated with mutant MLK3, and they support the functional role of mutant MLK3 by showing a deregulation of several signaling pathways known to play important roles in the development and progression of colorectal cancer. The results also suggest that mutant MLK3 may be a novel modulator of WNT signaling, and pinpoint the activation of PCP pathway as a possible mechanism underlying the invasive potential of MLK3 mutant cells.

Keywords: Colorectal cancer, MLK3, WNT pathway, MSI, Planar cell polarity

\section{Background}

Mixed-lineage kinase 3 (MLK3) belongs to a family of seven different mammalian MLKs, that are clustered in three subgroups accordingly with their structural similarities: the MLKs (MLK1, MLK2, MLK3, MLK4); the dual-leucine-zipper-bearing kinases (Dlk and Lzk); and zipper sterile- $\alpha$-motif kinase (Zak $\alpha$ and Zak $\beta$ ) [1].

MLK3 protein is composed by a Src-homology-3 (SH3) domain, located at the amino terminus of the protein,

\footnotetext{
*Correspondence: rseruca@ipatimup.pt

'Instituto de Patologia e Imunologia Molecular da Universidade do Porto, Rua Dr. Roberto Frias, 4200-465 Porto, Portugal

${ }^{5}$ Medical Faculty of the University of Porto, Porto, Portugal

Full list of author information is available at the end of the article
}

followed by a kinase domain, a leucine-zipper region, a Cdc42/RAC interacting binding motif (CRIB) and a Proline/Serine/Threonine-rich (P/S/T-rich) carboxy terminal domain [1-4]. All these domains show a very high degree of homology between MLK members (MLK1-MLK4) [1], except the carboxy-terminus $\mathrm{P} / \mathrm{S} / \mathrm{T}$ - rich domain which is the less conserved region among MLK proteins [1].

MLK3 is a serine/threonine protein kinase that regulates the mitogen-activated protein kinase (MAPK) pathway, activating ERK, p38 and JNK in response to extracellular signals $[1,5]$. Additionally, functional studies have demonstrated that overexpression of wildtype MLK3 leads to morphological transformation of 
NIH 3T3 fibroblasts and growth in soft agar, a capacity that is MEK/ERK dependent [6]. Further, MLK3 has been demonstrated to function as a scaffolding protein, involved in the formation of a multiprotein complex containing MLK3/BRAF/RAF1 $[1,5,7,8]$. The formation of this complex was shown to be important for the activation of wild-type BRAF and, consequently, to the activation of ERK signaling [1,5,7,8]. Furthermore, MLK3 was reported to be important for the proliferation of tumor cells, bearing either oncogenic KRAS or neurofibromatosis-1 (NF1) or NF2 inactivating mutations [8]. In addition, the MLK3 signaling activation was associated with an increase in the migratory and invasive capacity of tumor cells in gastric [9], breast [10-12], lung [13] and ovarian [14] cancers. All together, these observations implicate MLK3 as a cancerrelated gene although, until recently, nothing was known about MLK3 gene deregulation in primary cancer tissues.

Our group reported the occurrence of MLK3 mutations in microsatellite unstable (MSI) gastrointestinal tumors (both sporadic and hereditary forms) in a frequency of about $20 \%$. Using in vitro transforming assays, we demonstrated that several MLK3 mutations affecting different domains of the protein had transforming potential when compared to cells expressing the wild-type and the kinasedead forms of the protein [15]. These results were further supported by in vivo studies in which one of the two most transforming mutations $(\mathrm{P} 252 \mathrm{H}$ - located in the kinase domain) was found to be tumorigenic and to give rise to highly invasive tumors when subcutaneously injected in nude mice. Thus, our previous work pointed mutant MLK3 as a new oncogene in MSI gastrointestinal cancers, however, the signaling pathways associated to its oncogenic activity remained to be explored.

In this work, we aimed at identifying the signaling pathways associated to mutant MLK3, in particular the P252H mutation. The reasons underlying the choice for this mutation were: (a) it was one of the most transforming mutations previously analyzed; (b) showed high tumorigenic capacity with strong invasive potential in in vivo studies; and (c) it was located in the kinase domain which is an important domain for the regulation of downstream signaling pathways.

The results showed that $\mathrm{P} 252 \mathrm{H}$ mutation interferes with important colorectal cancer-associated signaling pathways such as WNT, MAPK, NOTCH, TGF- $\beta$ and P53.

\section{Methods}

\section{cDNA constructs and mutagenesis}

Wild-type MLK3 and mutant sequences were cloned into pLENTID6/V5 directional TOPO (Invitrogen). Mutant MLK3 P252H sequence was generated by site-directed mutagenesis using the MLK3 wild-type sequence cloned in pLENTID6/V5 as template. pLENTID6/V5 empty vector
(Mock) was obtained by the insertion of a small fragment of cDNA in order to circularize the plasmid.

\section{Cell lines}

Human HEK293 were maintained in DMEM (Gibco, Invitrogen) supplemented with $10 \% \mathrm{FBS}$ and $1 \%$ penicillin-streptomycin (Gibco, Invitrogen), and were incubated in a humidified chamber with $5 \% \mathrm{CO}_{2}$ at $37^{\circ} \mathrm{C}$.

\section{Transfections}

For HEK293 stable transfections, ViraPower Lentiviral Expression kit (Invitrogen) was used for the transduction of the MLK3 wild-type and mutant P252H sequences as well as the empty vector. Lentiviral transduction was performed following the manufacturer's instructions. Transduced cells were selected by antibiotic resistance (blasticidin, $12 \mu \mathrm{g} / \mathrm{ml}$ ) (Gibco, Invitrogen). The expression levels of MLK3 in the different clones selected were measured by western-blot.

\section{RNA extraction and CDNA synthesis}

Total RNA was isolated from cell lines using TriPure Isolation Reagent (Roche Applied Science), following manufacturer's instructions. Complementary DNA was synthesized from $1 \mu \mathrm{g}$ of total RNA using SuperScriptII Reverse Transcriptase (Invitrogen) and Random Primers (Invitrogen).

\section{Labeling and hybridization}

Five hundred ng aliquots of RNA from the samples were quality checked using the Agilent 2100 Byoanalyzer and only samples above integrity quality number (RIN) 8 [16] were used and amplified according to the specifications of the Illumina ${ }^{\circ}$ TotalPrep ${ }^{\text {Tw }}$ RNA Amplification Kit (Ambion, Austin, TX, USA). The cRNA samples were applied to the arrays of Sentrix ${ }^{\odot}$ Human-v6 Expression BeadChip (Illumina, San Diego, CA, USA) and hybridized according to manufacturer's specifications. The Sentrix BeadChips were scanned with the Illumina's Beadarray system 500G Scanner (Illumina').

\section{Microarray data analysis}

The signal intensity was extracted from the hybridization images, background subtracted and normalized using Illumina Inc. BeadStudio software version 3.3.7. The data produced was checked against the Illumina internal quality controls and loaded into the Bioconductor software $[17,18]$. To identify differentially expressed genes based on a moderate $t$-test, the bioconductor Limma package [19] was used. Genes were selected based on a $p$-value cut-off (after adjustment) of $p<0.01$ to control the false discovery rate (FDR) [20]. To test the association of selected differentially expressed genes with KEGG pathways [21], information provided in the Illumina annotation files 


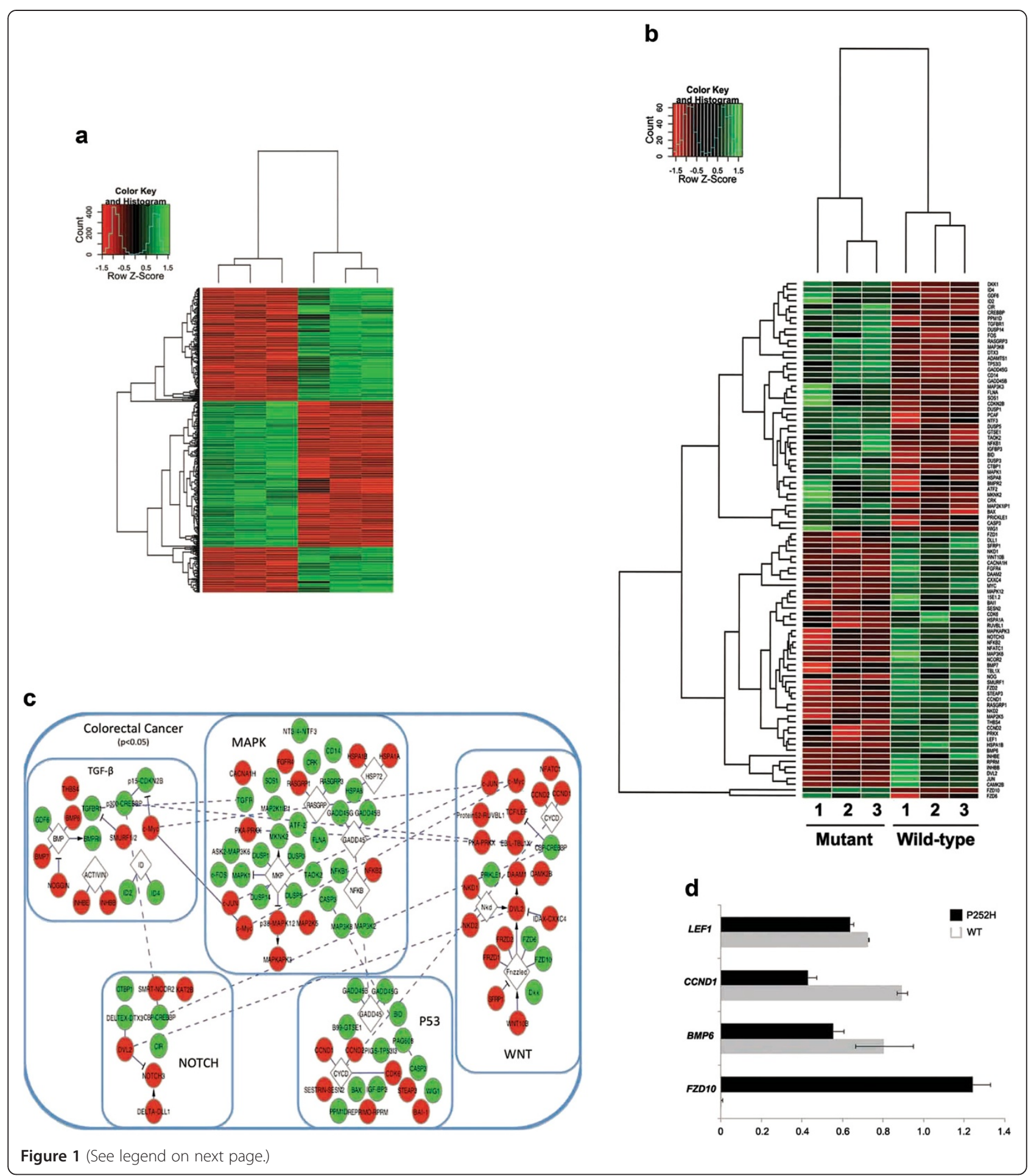


(See figure on previous page.)

Figure 1 Expression profiling of biological triplicates of HEK293 cells transfected with mutant and wild-type MLK3. (a) Heatmap of the 445 genes which clearly classify the mutant vs the wild-type, selected on the basis of a 2 log-fold change in expression between cells transfected with $\mathrm{P} 252 \mathrm{H}$ mutant and wild-type forms of MLK3, and eliminating genes which were differentially expressed between the Mock and wild-type transfection (red=down-regulated in P252H mutant MLK3, green=up-regulated in P252H mutant MLK3). (b) Illustrates a subset of the heatmap, focusing on genes which are part of the colorectal cancer pathway which is significantly affected ( $p$-value $=0.03$ ). (c) Graphical representation of the colorectal pathway (larger rounded rectangle) and relevant pathways contained within it (smaller rounded rectangles), such as MAPK, WNT, TGF- $\beta$, NOTCH and p53, indicating differentially expressed genes (ovals) present in Figure 1, their relationships to each other (solid line indicates a direct protein-protein interaction, T shaped ending for inhibition interactions, arrow ending for activation interactions, and no ending for other known interactions), as well as the direction of the expression change (red = down-regulated in P252H mutant MLK3 transfection, green = up-regulated in mutant MLK3). White Diamond shaped boxes indicate entire gene families, which are significantly affected, genes within the families are shown as colored ovals. Long dashed lines indicate genes, which are present in multiple pathways. (d) Graphical representation of Real-time PCR quantification of mutant vs wild-type MLK3 targets (LEF1, CCND1, BMP6 and FZD10) selected from expression microarrays.

(Sentrix ${ }^{\oplus}$ Human-v6 Expression BeadChip) Hypergeometric test available in the GOstats packages [22] was used. A $p$-value cut off of 0.05 was considered. Microarray data can be found at the GEO repository with the accession number GSE54611.

\section{Real-time PCR}

One $\mu \mathrm{l}$ of cDNA was added to $10 \mu \mathrm{l}$ Real-Time PCR mixtures containing 1x TaqMan ${ }^{\oplus}$ Universal PCR Master Mix, No AmpErase ${ }^{\circledR}$ UNG (Applied Biosystems) and 1x $\operatorname{TaqMan}^{\circ}$ MGB specific probes and primers mix. Taqman expression assays for BMP6 (Hs01099594_ m1), CCND1 (Hs00277039_m1), FZD10 (Hs00273077_ s1), LEF1 (Hs00212390_m1) were purchased from Applied Biosystems. The eukaryotic 18S rRNA assay (Hs99999901_s1; Applied Biosystems) was used as an endogenous control gene. Standard TaqMan thermocycling conditions were used: 1 cycle of 2 minutes at $50^{\circ} \mathrm{C}, 1$ cycle of 10 minutes at $95^{\circ} \mathrm{C}, 40$ cycles of $15 \mathrm{sec}-$ onds at $95^{\circ} \mathrm{C}$ followed by 1 minute at $60^{\circ} \mathrm{C}$ in an $\mathrm{ABI}$ Prism 7000 (Applied Biosystyems). Real-Time PCR assays (absolute quantification) were performed in, at least, three biological replicas.

\section{Statistical analyses}

For statistical analyzes of in vitro transformation assays a $t$-student test was used and $p<0.05$ was taken as statistically significant. Specific statistical tests used for microarray interpretation are embedded in the corresponding materials and methods section.

\section{Results and discussion}

MLK3 P252H mutation affects fundamental colorectal cancer-associated pathways

In order to assess the effect of a tumorigenic MLK3 mutant on a genome-wide level, we performed microarray-based

Table 1 KEGG Pathways significantly affected by mutant MLK3

\begin{tabular}{|c|c|c|c|c|c|c|}
\hline $\begin{array}{l}\text { KEGG } \\
\text { ID }\end{array}$ & p-value & $\begin{array}{l}\text { Odds } \\
\text { Ratio }\end{array}$ & Exp count & Count & Size & Term \\
\hline 970 & 0.000 & 4.669 & 5 & 16 & 35 & Aminoacyl-tRNA biosynthesis \\
\hline 290 & 0.001 & 7.682 & 2 & 7 & 12 & Valine, leucine and isoleucine biosynthesis \\
\hline 5120 & 0.001 & 2.477 & 11 & 21 & 68 & Epithelial cell signaling in Helicobacter pylori infection \\
\hline 251 & 0.004 & 3.184 & 5 & 11 & 30 & Glutamate metabolism \\
\hline 630 & 0.009 & 4.693 & 2 & 6 & 13 & Glyoxylate and dicarboxylate metabolism \\
\hline 4115 & 0.013 & 1.984 & 11 & 18 & 68 & p53 signaling pathway \\
\hline 330 & 0.014 & 2.517 & 5 & 11 & 35 & Arginine and proline metabolism \\
\hline 3020 & 0.018 & 2.923 & 4 & 8 & 23 & RNA polymerase \\
\hline 5020 & 0.026 & 2.948 & 3 & 7 & 20 & Parkinson's disease \\
\hline 670 & 0.027 & 3.283 & 2 & 6 & 16 & One carbon pool by folate \\
\hline 5210 & 0.03 & 1.721 & 13 & 20 & 84 & Colorectal cancer \\
\hline 600 & 0.031 & 2.155 & 6 & 11 & 39 & Sphingolipid metabolism \\
\hline 260 & 0.038 & 1.995 & 7 & 12 & 45 & Glycine, serine and threonine metabolism \\
\hline 5040 & 0.041 & 2.241 & 5 & 9 & 31 & Huntington's disease \\
\hline 5110 & 0.044 & 2.01 & 6 & 11 & 41 & Cholera - infection \\
\hline
\end{tabular}


expression profiling experiments. We used Illumina Whole Genome arrays on three biological replicas of human HEK293 cells stably transfected with the wild-type MLK3, with the MLK3 P252H mutation and with the empty vector (Mock). The expression profiles were obtained by comparing all biological replicas from each transfection experiment. Colorectal cancer cell lines were not used in this experiment, as proteins from the MAPK pathway are frequent mutation targets in this type of cancer, and would most likely interfere with the interpretation of the results. The expression profiles were compared to identify genes that were differentially expressed at least 2 log-fold (FDR < 0.01 ) between wild-type and Mock, as well as between $\mathrm{P} 252 \mathrm{H}$ and Mock. A final set of 445 genes was identified which showed significant differential expression only between $\mathrm{P} 252 \mathrm{H}$ and wild-type and not between wild-type and Mock (Figure 1a). The most statistically significantly differentially expressed genes are displayed in Figure 1b. The genes identified were significantly enriched $(p<0.05)$ in several KEGG pathways (Table 1) involved in overall biosynthesis processes, as well as in response in disease relevant processes. Interestingly, the colorectal pathway, which encompasses several relevant pathways such as WNT, MAPK, NOTCH, TGF- $\beta$ and P53, was significantly over-represented (Figure 1c). These signaling pathways are crucial to maintain intestinal epithelium homeostasis by balancing the rate of proliferation, apoptosis, and differentiation along the crypt-villus axis, and their de-regulation is commonly associated to colorectal cancer initiation and progression [23]. Corroborating our results, a recent study using both in vivo and in vitro approaches showed that MLK3 signaling is important in intestinal mucosal healing and epithelial cell motility [24], therefore implicating MLK3 signaling in the maintenance of intestinal epithelial homeostasis.

In order to further validate the microarray data, a set of differentially expressed genes (LEF1, CCND1, FZD10, and BMP6) were selected for validation by real-time PCR in HEK293 cells stably expressing MLK3 wild-type or MLK3 P252H. The results obtained with the microarrays were validated for all genes tested (Figure 1d).

Of particular interest are the alterations that mutant MLK3 induces in the WNT pathway. Activation of canonical WNT signaling through WNT/ $\beta$-catenin cascade has traditionally been regarded as a critical player in colorectal tumorigenesis [25]. More recently, accumulating evidence supports a role for the non-canonical WNT planar cell polarity (PCP) pathway, a signaling cascade involved in the polarization of cells during tissue remodeling, and cell adhesion and motility, in cancer progression, invasion, metastasis, and angiogenesis [26-28]. A more detailed analyzes of our microarray data showed that the expression of several molecular components of the canonical pathway, such as DVL2,
LEF1, CCND1 and c-MYC were down-regulated in MLK3 mutant cells, and the expression of DKK, a wellknown negative regulator of canonical WNT signaling [29], was up-regulated (Figure 1c and d). On the other hand, genes encoding two WNT receptors known to act as negative regulators of the canonical $\mathrm{WNT} / \beta$-catenin signaling cascade and as positive regulators of the PCP pathway, FZD6 and FZD10, were found to be up-regulated in P252H cells (Figure $1 \mathrm{c}$ and $\mathrm{d}$ ). Taken together, the down-regulation of DVL2, LEF1, CCND1 and c-MYC, and the up-regulation of DKK and FZD receptors suggest a role of mutant MLK3 as a molecular switch between canonic and non-canonic WNT signaling. In accordance, it was recently reported that MLK3 reduces the expression of $\beta$-catenin/TCF downstream targets by promoting the interaction between $\beta$ catenin and KLF4, a known repressor of $\beta$-catenin/TCF transcriptional activity [30]. Furthermore, in accordance with a role of PCP in colorectal cancer, FZD10 was recently demonstrated to be up-regulated in colorectal cancers and matched liver metastases, and its overexpression was associated with the activation of noncanonical WNT pathway $[31,32]$.

\section{Conclusion}

In conclusion, our results provide an overall view of the expression profile associated with mutant MLK3, and they support the functional role of mutant MLK3 by showing a deregulation of several signaling pathways known to play important roles in the development and progression of colorectal cancer. The results also suggest that mutant MLK3 may be a novel modulator of WNT signaling, and pinpoint the activation of the PCP pathway as a possible mechanism underlying the invasive potential of MLK3 mutant cells. Nevertheless, further studies are required in order to validate this hypothesis in a panel of gastrointestinal cell lines and human primary tumors, to determine if the altered signaling pathways are common to other MLK3 mutations, and to investigate the role of mutant MLK3 in the context of mutant KRAS and BRAF genes.

\footnotetext{
Abbreviations

MLK: Mixed lineage kinase; WNT: Wingless type; BMP: Bone morphogenetic protein; TGF- $\beta$ : Transforming growth factor $\beta$; MSI: Microsatellite instability; PCP: Planar cell polarity; DKK: Dickkopf; FZD: Frizelled; DVL2: Dishevelled;

LEF1: Lymphoid enhancer binding factor 1; CCND1: Cyclin D1; KLF4: Krupfellike factor; c-MYC: v-myc avian myelocytomatosis viral oncogene homolog; TCF: T cell-specific transcription factor; PCR: Polymerase chain reaction; MEK: MAP kinase-ERK kinase; ERK: Extracellular signal-regulated kinase; MAPK: Mitogen-activated protein kinase; JNK: c-Jun amino-terminal kinase; BRAF: v-raf murine sarcoma viral oncogene homolog B; KRAS: Kirsten rat sarcoma viral oncogene homolog; Dlk: Dual-leucine-zipper-bearing kinase; Lzk: Leucine-zipper-bearing kinase; Zak: Zipper sterile-a-motif kinase; SH3: Src-homology-3.
}

\section{Competing interests}

The authors declare that they have no competing interests. 


\section{Authors' contributions}

SV contributed to the acquisition, analysis and interpretation of data and drafted the manuscript; AP participated in the acquisition, analysis and interpretation of data; DL contributed to the acquisition, analysis and interpretation of data; MJO was responsible for the conception and design of the experimental system and critically reviewed the manuscript; FS was involved in the acquisition, analysis and interpretation of data; ES contributed for the conception and design of the experimental system and critically reviewed the manuscript; RS contributed to the conception and design of the project and experimental system, interpretation of data, and was responsible for the final approval of the version to be published.

\section{Acknowledgements}

This work was supported by Grants from The Portuguese Foundation for Science and Technology (FCT) (Project PTDC/SAU-OBD/68310/2006), the Portuguese Ministry for Science and Education, by FEDER- European Fund for the Regional Development and the Programs COMPETE- Programa Operacional de Fatores de Competitividade (POFC) and PEst-C/SAU/LA0002/ 2013. MJ Oliveira was supported by a Investigator FCT Grant and SV by a Post-doctoral fellowship (SFRH/BPD/69089/2010) from the Portuguese Foundation for Science and Technology (FCT).

\section{Author details}

${ }^{1}$ Instituto de Patologia e Imunologia Molecular da Universidade do Porto, Rua Dr. Roberto Frias, 4200-465 Porto, Portugal. ${ }^{2}$ New Therapies Group, INEB-Institute for Biomedical Engineering, Porto, Portugal. ${ }^{3}$ CBM S.c.r.l. AREA SCIENCE PARK, Trieste, Italy. ${ }^{4}$ Center for Translational Genomics and Bioinformatics, San Raffaele Scientific Institute, Milan, Italy. ${ }^{5}$ Medical Faculty of the University of Porto, Porto, Portugal.

Received: 23 September 2013 Accepted: 25 February 2014 Published: 14 March 2014

\section{References}

1. Gallo KA, Johnson GL: Mixed-lineage kinase control of JNK and p38 MAPK pathways. Nat Rev Mol Cell Biol 2002, 3(9):663-672.

2. Burbelo PD, Drechsel D, Hall A: A conserved binding motif defines numerous candidate target proteins for both Cdc42 and Rac GTPases. J Biol Chem 1995, 270(49):29071-29074

3. Gallo KA, Mark MR, Scadden DT, Wang Z, Gu Q, Godowski PJ: Identification and characterization of SPRK, a novel src-homology 3 domaincontaining proline-rich kinase with serine/threonine kinase activity. J Biol Chem 1994, 269(21):15092-15100.

4. Ing Y, Leung I, Heng H, Tsui L, Lassam N: MLK-3: identification of a widelyexpressed protein kinase bearing an $\mathrm{SH} 3$ domain and a leucine zipper-basic region domain. Oncogene 1994, 9(6):1745-1750.

5. Chadee D, Kyriakis J: A novel role for mixed lineage kinase 3 (MLK3) in BRaf activation and cell proliferation. Cell Cycle 2004, 3(10):1227-1229.

6. Hartkamp J, Troppmair J, Rapp UR: The JNK/SAPK activator mixed lineage kinase 3 (MLK3) transforms NIH 3T3 cells in a MEK-dependent fashion. Cancer Res 1999, 59(9):2195-2202.

7. Chadee DN, Kyriakis JM: MLK3 is required for mitogen activation of B-Raf, ERK and cell proliferation. Nat Cell Biol 2004, 6(8):770-776.

8. Chadee DN, Xu D, Hung G, Andalibi A, Lim DJ, Luo Z, Gutmann DH, Kyriakis JM: Mixed-lineage kinase 3 regulates B-Raf through maintenance of the B-Raf/Raf-1 complex and inhibition by the NF2 tumor suppressor protein. Proc Natl Acad Sci U S A 2006, 103(12):4463-4468.

9. Mishra P, Senthivinayagam S, Rangasamy V, Sondarva G, Rana B: Mixed lineage kinase-3/JNK1 axis promotes migration of human gastric cancer cells following gastrin stimulation. Mol Endocrinol 2010, 24(3):598-607.

10. Chen J, Gallo KA: MLK3 regulates paxillin phosphorylation in chemokinemediated breast cancer cell migration and invasion to drive metastasis. Cancer Res 2012, 72(16):4130-4140.

11. Chen J, Miller EM, Gallo KA: MLK3 is critical for breast cancer cell migration and promotes a malignant phenotype in mammary epithelial cells. Oncogene 2010, 29(31):4399-4411.

12. Cronan MR, Nakamura K, Johnson NL, Granger DA, Cuevas BD, Wang JG, Mackman N, Scott JE, Dohlman HG, Johnson GL: Defining MAP3 kinases required for MDA-MB-231 cell tumor growth and metastasis. Oncogene 2012, 31(34):3889-3900.
13. Chien ST, Lin SS, Wang CK, Lee YB, Chen KS, Fong Y, Shih YW: Acacetin inhibits the invasion and migration of human non-small cell lung cancer A549 cells by suppressing the p38alpha MAPK signaling pathway. Mol Cell Biochem 2011, 350(1-2):135-148.

14. Zhan Y, Abi Saab WF, Modi N, Stewart AM, Liu J, Chadee DN: Mixed lineage kinase 3 is required for matrix metalloproteinase expression and invasion in ovarian cancer cells. Exp Cell Res 2012, 318(14):1641-1648.

15. Velho S, Oliveira C, Paredes J, Sousa S, Leite M, Matos P, Milanezi F, Ribeiro AS, Mendes N, Licastro D, Karhu A, Oliveira MJ, Ligtenberg M, Hamelin R, Carneiro F, Lindblom A, Peltomaki P, Castedo S, Schwartz S Jr, Jordan P, Aaltonen LA, Hofstra RM, Suriano G, Stupka E, Fialho AM, Seruca R: Mixed lineage kinase 3 gene mutations in mismatch repair deficient gastrointestinal tumours. Hum Mol Genet 2010, 19(4):697-706.

16. Schroeder A, Mueller O, Stocker S, Salowsky R, Leiber M, Gassmann M, Lightfoot S, Menzel W, Granzow M, Ragg T: The RIN: an RNA integrity number for assigning integrity values to RNA measurements. BMC Mol Biol 2006, 7(1):3.

17. Gentleman R, Carey V, Bates D, Bolstad B, Dettling M, Dudoit S, Ellis B, Gautier L, Ge Y, Gentry J, Hornik K, Hothorn T, Huber W, lacus S, Irizarry R, Leisch F, Li C, Maechler M, Rossini A, Sawitzki G, Smith C, Smyth G, Tierney L, Yang J, Zhang J: Bioconductor: open software development for computational biology and bioinformatics. Genome Biol 2004, 5(10):R80.

18. R Development Core Team: $R$ : A language and environment for statistical computing. Vienna, Austria: R Foundation for Statistical Computing; 2008

19. Smyth G: Limma: linear models for microarray data. New York: Springer; 2005.

20. Benjamini $Y$, Hochberg $Y$ : Controlling the false discovery rate: a practical and powerful approach to multiple testing. J R Stat Soc B 1995, 57:289-300.

21. Liu T, Lin C, Falcon S, Zhang J, MacDonald J: Kegg: a data package containing annotation data for KEGG. R package version 2.2.0. 2007

22. Gentleman R, Falcon S: Gostats: tools for manipulating GO and microarrays. $\mathrm{R}$ package version 2.6.0. 2007:

23. Radtke F, Clevers H, Riccio O: From gut homeostasis to cancer. Curr Mol Med 2006, 6(3):275-289.

24. Kovalenko PL, Kunovska L, Chen J, Gallo KA, Basson MD: Loss of MLK3 signaling impedes ulcer healing by modulating MAPK signaling in mouse intestinal mucosa. Am J Physiol Gastrointest Liver Physiol 2012, 303(8):G951-G960.

25. de Lau W, Barker N, Clevers H: WNT signaling in the normal intestine and colorectal cancer. Front Biosci 2007, 12:471-491.

26. Naz G, Pasternack SM, Perrin C, Mattheisen M, Refke M, Khan S, Gul A, Simons M, Ahmad W, Betz RC: FZD6 encoding the Wnt receptor frizzled 6 is mutated in autosomal-recessive nail dysplasia. $\mathrm{Br} J$ Dermatol 2012 166(5):1088-1094.

27. Seifert JR, Mlodzik M: Frizzled/PCP signalling: a conserved mechanism regulating cell polarity and directed motility. Nat Rev Genet 2007, 8(2):126-138.

28. Wang Y: Wnt/Planar cell polarity signaling: a new paradigm for cance therapy. Mol Cancer Therap 2009, 8(8):2103-2109.

29. Rao TP, Kuhl M: An updated overview on Wnt signaling pathways: a prelude for more. Circ Res 2010, 106(12):1798-1806.

30. Thylur RP, Senthivinayagam S, Campbell EM, Rangasamy V, Thorenoor N Sondarva G, Mehrotra S, Mishra P, Zook E, Le PT, Rana B: Mixed lineage kinase 3 modulates beta-catenin signaling in cancer cells. J Biol Chem 2011, 286(43):37470-37482

31. Fukukawa C, Nagayama S, Tsunoda T, Toguchida J, Nakamura Y, Katagiri T: Activation of the non-canonical Dvl-Rac1-JNK pathway by Frizzled homologue 10 in human synovial sarcoma. Oncogene 2009, 28(8):1110-1120.

32. Nagayama S, Yamada E, Kohno Y, Aoyama T, Fukukawa C, Kubo H, Watanabe G, Katagiri T, Nakamura Y, Sakai Y, Toguchida J: Inverse correlation of the up-regulation of FZD10 expression and the activation of beta-catenin in synchronous colorectal tumors. Cancer Sci 2009, 100(3):405-412.

doi:10.1186/1471-2407-14-182

Cite this article as: Velho et al:: Dissecting the signaling pathways associated with the oncogenic activity of MLK3 P252H mutation. BMC Cancer 2014 14:182 